\title{
FTU Wireless Intelligent Information Acquisition System Based on ARM11
}

\author{
Xie Zhiyuan ${ }^{1, a}$, Liu Jianning ${ }^{1, b}$, Xue Yujuan ${ }^{1, c}$ \\ North China Electric Power University, Yong hua bei da jie 619, Baoding, Hebei, China \\ aemail:zhiyuanxie@263.net \\ bemail:liujianning_9@163.com \\ cemail:xueyujuan0@163.com
}

Keywords: ARM11; Windows CE; serial driver; FTU; intelligent information acquisition

\begin{abstract}
With the development of smart grid construction, a large number of intelligent electrical equipment has been applied. In order to facilitate the onsite FTU data acquisition, use S3C6410 with ARM11 kernel and Windows CE embedded system as platform to develop and design wireless intelligent information acquisition system. After the hardware platform and embedded system are completed, add wireless module driver, develop information frame format of communication protocol, and according to the application software requirements, design and develop FTU wireless communication applications. After experimental testing, FTU wireless communications is fulfilled between the portable equipment and $10 \mathrm{KV}$ power lines; then acquires and process the returning data. This article provides feasibility program for the FTU field local test.
\end{abstract}

\section{Introductions}

Currently $10 \mathrm{kV}$ overhead line has broad coverage and high tripping rate, and serious shortage of switch on column ${ }^{[1]}$, with the continuous development of our intelligent power grid construction, feeder terminal unit (FTU) and other field monitoring terminals have been increasingly applied ${ }^{[2]}$. FTU is the interface of automation system and device connection, and it can communicate with distribution station, provide related information of running status of power distribution and control information ${ }^{[3]}$, and it can also receive the control command from distribution substation to control and regulate power distribution equipment.

Although FTU has perfect communication control device, and it can realize two-way communication and information exchange with communication main station, but when the remote communication occurs fault, local test is needed for the device, because FTU is mounted on $10 \mathrm{kV}$ circuit, it brings much inconvenience for test. Embedded system has characteristics such as low voltage, low power, high integration, rich hardware resources, high processing speed ${ }^{[4]}$, therefore this article will research wireless communication with FTU based on portable device with ARM11 embedded Windows CE operating system to complete local data test.

\section{Wireless intelligent information acquisition system platform}

Hardware platform. Main hardware is Samsung's S3C6410 based on ARM11 framework as processor, resistance type touch screen, and RF4432D20 wireless module.

The major hardware are with ARM11's S3C6410 as the core, and S3C6410 uses ARM1176JZF-S kernel, which is a 16/32bit RISC microprocessor with high performance and low power, it is a perfect external equipment, supporting TFT 24Bit LCD screen, at the same time have operating frequency as high as $667 \mathrm{MHz}{ }^{[5]}$.The touch screen has display and operation section; DDR and Flash are memory and storage for running ARM11 and WINCE operating system; SD card is used for storing the application, data and the system event log; wireless module uses RF4432D20 to have wireless communication with FTU. System hardware diagram is shown in Figure 1: 


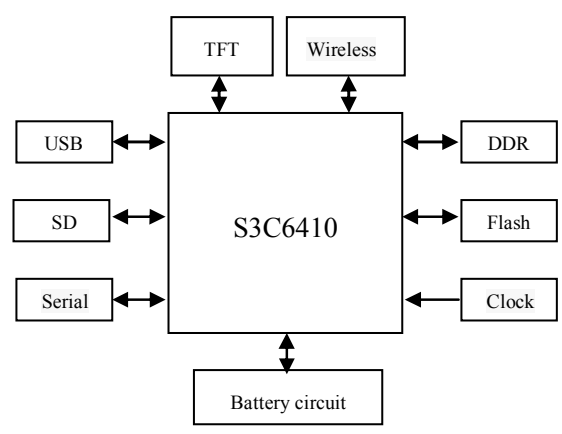

Fig.1 Diagram of hardware part

WINCE Operating System. Windows CE system is a 32 bit embedded operating system introduced by Microsoft. It has good modularity, good multimedia functions and friendly man-machine interface ${ }^{[6]}$. Windows $\mathrm{CE}$ is designed to be highly modular, good real-time and strong communication capability, and it can support a variety of embedded operating system of CPU.

This study focuses on the operating system version Windows CE 6.0 of embedded ARM11. Unlike the previous version, the redesigned core supports 32,000 concurrent processes, and each process has a $2 \mathrm{~GB}$ virtual memory addressing space, and it also can maintain the system's real-time response $^{[7]}$.

Windows CE system structure is divided into hardware layer, OEM layer, operating system layer and application layer, the four layers closely and mutually cooperate, then obtain user needs from the application and apply them to the hardware operation ${ }^{[8]}$.

Research focuses on adding driver to the wireless module RF4432D20, and this belongs to OEM layer, its purpose is to perform hardware sampling and provide uniform interface to facilitate operating system for wireless module operation. While the FTU application software is the application layer on the top, and the application uses Win32 API provided by operating system to perform system call base interactive, and finally control hardware through the driver.

\section{Software development and test}

Windows CE 6.0 system is embedded on the hardware platform, wireless intelligent information acquisition system is based on it to add wireless module driver, develop FTU communication software, and test FTU communication to get the relevant data.

Add wireless module driver. RF4432D20 wireless module provides serial TTL, 232 and 485 interface, through asynchronous serial port UART to carry on the control, the module is directly connected to ARM11, and use TTL interface, the connection diagram is as shown in Figure 2:

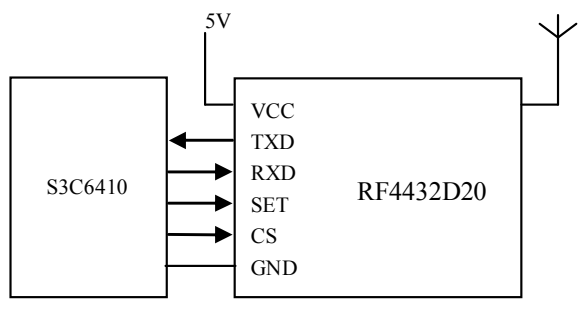

Fig.2 Diagram of Wireless module connection

From figure 2, we can see that there are six pins in the wireless module, of which only TXD and RXD pins are for data transmission, and the pin functions are shown as in Table 1: 
Tab.1 Description of Wireless module pins

\begin{tabular}{|l|l|}
\hline Pins & Description \\
\hline VCC & Connected with the positive pole of the power supply \\
\hline TXD & Send serial data port \\
\hline RXD & Serial receive data port \\
\hline SET & Enable configuration parameters \\
\hline CS & Enable module \\
\hline GND & Power ground \\
\hline
\end{tabular}

From table 1, we can see the function of each pin, and wireless module does not need to be configured again, and does not need to use SET pins, while TXD, RXD and CS as the control pins, need to be connected with S3C6410, and they are controlled by driver.

Wireless module controls its data reading and writing through serial ports, and it is equal to the adding of corresponding serial port driver at the bottom layer in the system, which belongs to the stream interface driver. Stream drive is an export flow interface which can be chosen by any peripheral devices driver with producing or consuming data as the main function, such as serial device driver is a typical stream interface driver.

If we classify serial port driver by driving structure, it belongs to layered driver. Layered driver divides driver code into the upper layer of Model Device Driver (MDD) and the lower layer of Platform Dependent Driver (PDD). The MDD layer contains the common codes for all the given types of drivers, and the PDD layer is consisted of codes specific to given hardware device or platform. Driven development generally only needs to write PDD layer codes specific to the hardware platform. Hierarchical driver structure is as shown in Figure 3:

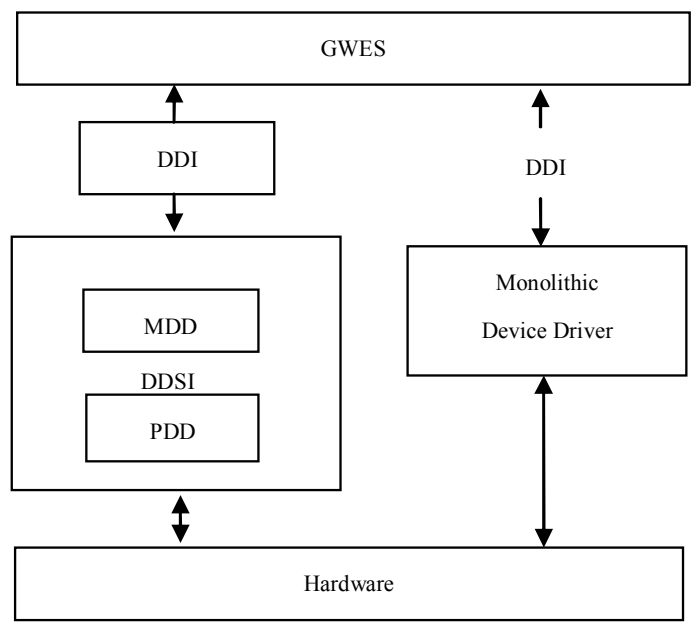

Fig.3 Structure of layered driver

As shown in Figure 3, the device driver interface DDI is the functions set in the MDD, and it is called by GWES. The DDSI function is the functions set in the PDD, and it is called by MDD. MDD accomplishes the link to layer PDD and defines its desired function, and derives the DDI function to operating system, and it also processes complex tasks such as interrupt ${ }^{[9]}$.

In order to create a serial port driver, we first need to create a dynamic link library which contains the necessary entrance points for driver, the stream interface functions are also known as stream interface which defines serial for the functions from this dynamic link library (DLL) files, and its name is serial.def, it is used to illustrate which functions are derived from the dynamic link library, and the stream interface of driver is directly reflected here.

The program of wireless module driver will be registered and loaded in Windows CE. After Windows CE startup, system start system processes in succession, in which device.exe is responsible for loading current drive, and its settings is according to the registry information, then after loading the bus enumerator busenum.dll, bus enumerator starts enumerating every sub key of 
its own corresponding registry, looking for legitimate device driver in it. Therefore, to make the wireless module driver can be used normally, we need to add device registration information in registry. The registration information are as follows:

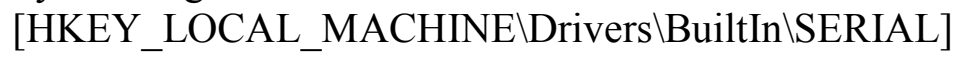

"DeviceĀrrayIndex" = dword:2

"Irq"=dword:18

"IoBase" = dword:50008000

"IoLen" = dword:2C

"Prefix"="COM"

"D11" = "SER6410.D11"

"Order"=dword:0

"Index"=dword:6

"Priority"=dword:0

"Port"="COM3"

"Device Type"=dword:0

"Friendly Name"="Serial Cable on COM3:"

After registering wireless module serial driver, by modifying platform.bib file, the driver will be added into the system. Drive file name should keep consistent with "Dll" key in registry.

The add contents are as follows:

Ser6410.dll \$(FLATRELEASEDIR) \ser6410.dll

NK SH

This shows that the wireless module drive is in the NK area of the image, and it will be loaded into memory assystem file. Recompile Windows CE system image, and download it to the hardware platform, then the drivers can be used.

Design of FTU communication software. Windows CE applications are developed using MFC class library of Microsoft Visual Studio $2005^{[10]}$. MFC is the class library provided by Microsoft, with class $\mathrm{C}++$ form to pack Windows API, and it contains an application framework which reduces the workload of developers.

Intelligent information acquisition system uses data frame format to perform wireless communication with FTU to improve the quality of communication. The data frame formats are shown in Figure 4 and Figure 5:

\begin{tabular}{|c|c|c|c|}
\hline EB & 90 & $\begin{array}{c}\text { high byte } \\
\text { of control }\end{array}$ & $\begin{array}{c}\text { low byte } \\
\text { of control }\end{array}$ \\
\hline $\begin{array}{c}\text { number } \\
\text { of target }\end{array}$ & $\begin{array}{c}\text { high byte } \\
\text { of data }\end{array}$ & $\begin{array}{c}\text { low byte } \\
\text { of data }\end{array}$ & CRC \\
\hline
\end{tabular}

Fig.4 Format of command frame

\begin{tabular}{|c|c|c|c|}
\hline EB & 90 & $\begin{array}{c}\text { high byte } \\
\text { of control }\end{array}$ & $\begin{array}{c}\text { low byte } \\
\text { of control }\end{array}$ \\
\hline $\begin{array}{c}\text { number } \\
\text { of target }\end{array}$ & $\begin{array}{c}8 \text { byte } \\
\text { of data }\end{array}$ & CRC & Mode \\
\hline
\end{tabular}

Fig.5 Format of response frame

FTU data perform transmission in wireless communication and in frame format as shown in corresponding Figure 5 and Figure 5. Information has basic unit as frame, and intelligent information acquisition system mainly transmits command frames, and each frame has 8 bytes; there are 16 bytes in the data frame of FTU returning intelligent information acquisition system, and each byte function is shown in Figure 5 and Figure 6.

Compile application software program according to the data frame, and the software flow chart is as shown in Figure 6: 


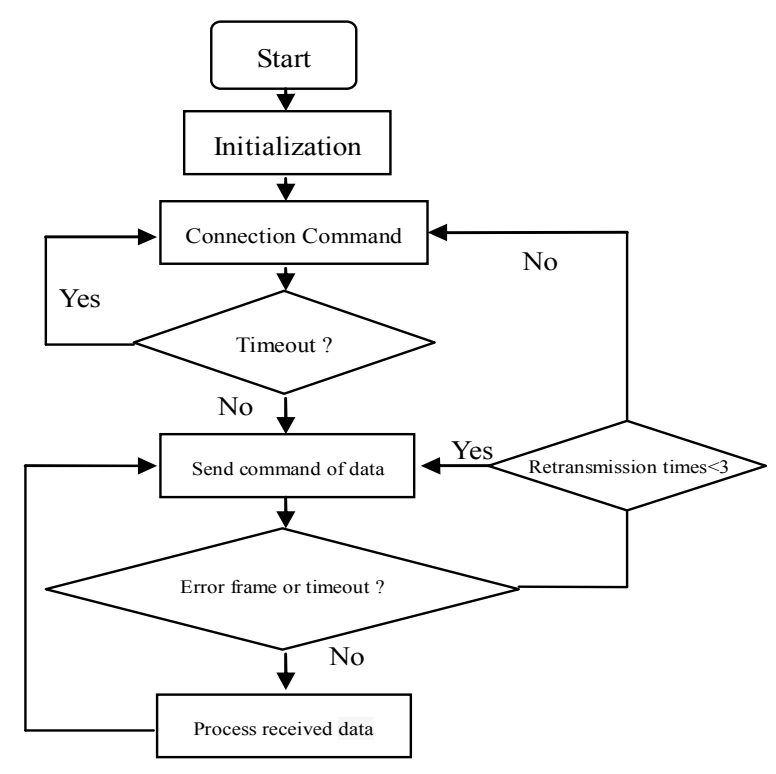

Fig.6 Flow chart of Software

From figure 6, we can see that after initialization of the application software of intelligent information acquisition system, it sends communication connection command to FTU, and waits for the responses of FTU response frame, if timeout, it will request connection once again, after successful connection, it will send data transfer command to control FTU and order it to return the related data parameters, if it is the error frame or timeout, then the order will be resent, when the retransmission orders are greater than or equal to 3, it will request reconnect.

After the application software design is completed, perform local test, and the software information interface's real-time display is as shown in Figure 7:

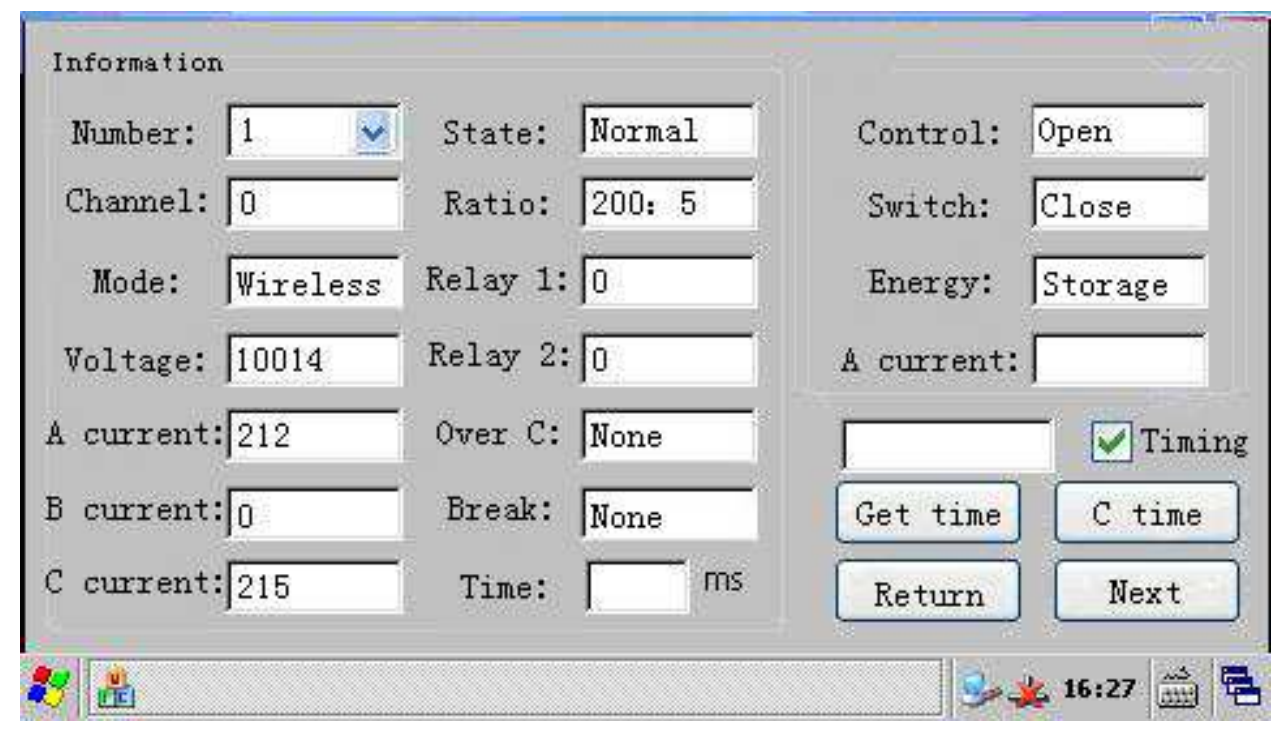

Fig.7 Interface of information

From figure 7, we can see that the portable intelligent information acquisition system is connected properly with FTU device, and receives and processes the returning data information; our tests successfully realize the local data collection for FTU wireless communication mode.

\section{Conclusions}

This article proposes intelligent information acquisition system designed and developed based on ARM11 and Windows CE platform for FTU equipment. When the construction personnel need to troubleshoot for 10KV FTU, they can use portable equipment and FTU wireless communication 
mode to control it and collect information. Through the test, this system is practically achieved, and it is convenient to perform onsite FTU information collection.

\section{References}

[1] ZHANG Yan-hui, ZHENG Dong-1iang, XIONG Wei. Discussion of 10kV feeder automation solutions[J]. Power System Protection and Control, 2010,38(16):150-152

[2] LUO Mei, YANG Hong-geng. An improved general matrix algorithm for fault locating in distribution system[J]. Power System Protection and Control, 2012,40(5):64-68

[3] PANG Jiyao. Application of Medium Speed Infrared Technique with Software Codec in FTU[J]. Modern Electronic Technique, 2010,3(314):200-204

[4] LIU Tie-dun, JI Jian-she, LI Chang-qing. Designing and realization of relay protection transceiver embedded monitor module[J]. Power System Protection and Control, 2011,39(17):126-129

[5] HU Shi-min. Design and Implement of Video Monitoring System Based on S3C6410[J]. Modern Electronic Technique, 2011,34(20):63-66

[6] LI Da-wei. Resolve Completely of Window CE Engineering Practice[M]. Beijing: China Electric Power Press, 2008.

[7] HE Zong-jian. Windows CE Embedded System[M]. Beijing: BeiHang University Press, 2006.

[8] JIANG Bo. Practice of Windows Embedded CE 6.0 Program Design [M]. Beijing: China Machine Press, 2009.

[9] ZHANG Dong-quan, TAN Nan-lin, SU Shu-qiang. Technology of Windows CE Practical Development(The Second Edition)[M]. Beijing: Publishing House of Electronics Industry, 2009.

[10]Zi-ying Wang. The design of scene simulation system based on MFC programming framework, Advanced Computer Control (ICACC), 2010 2nd International Conference on, 2010,3: 302 305 